\title{
Foreign body in tracheobronchial tree
}

\author{
Vikas Sinha · Rizwan Memon - Devang Gupta - Bela Prajapati · Vadisha Bhat · Yogesh More
}

\begin{abstract}
Inhalation of foreign body into the tracheobronchial tree is a medical emergency sometimes resulting into sudden death. The current mortality rate due to foreign body inhalation ranges from $0 \%$ to $1.8 \%$. Children with or without positive history of aspiration were examined and diagnosis was made on the basis of history, clinical findings, radiological evaluation and strong index of suspicion. A review of 30 cases of suspected foreign body aspiration revealed, children between 6 months were found to be very vulnerable to aspiration. Majority of children were boys. $80 \%$ of the patients had positive history of inhalation. Only $50 \%$ of the patients presented immediately i.e. within 24 hours after aspiration. Common symptoms were cough and respiratory distress. Decreased air entry was the significant clinical sign $(50 \%)$. Obstructive emphysema and mediastinal shift were found in the majority of cases (50\%). Rigid bronchoscopy under general anaesthesia and patient ventilating using a jet ventilator is a very safe and effective technique.
\end{abstract}

V. Sinha $(\bowtie) \cdot$ R. Memon ${ }^{2} \cdot$ D. Gupta ${ }^{3} \cdot$ Bela Prajapati $^{4}$.

V. Bhat ${ }^{5} \cdot$ Y. More ${ }^{6}$

${ }^{1}$ Professor and Head

${ }^{2}$ ENT Consultant

${ }^{3}$ Assistant Professor

${ }^{4}$ Assistant Professor

${ }^{5}$ Assistant Professor

${ }^{6}$ Resident

Department of ENT and Head and Neck Surgery

B. J. Medical College (Civil Hospital),

Ahmedabad,

Gujarat.

Professor and Head of Department

B.J.Medical College (Civil Hospital)

Ahmedabad,

Gujarat.

E mail: drsinhavikas@yahoo.co.in
Keywords Aspiration - Bronchoscopy - Tracheobronc hial foreign body

\section{Introduction}

Inhalation of foreign bodies into the tracheobronchial tree is a medical emergency, sometimes resulting into sudden death. More than three fourths of the cases of aspirated foreign bodies occur in children aged less than 3 years. Foreign body inhalation is usually accompanied by severe coughing, wheezing, dyspnoea or stridor. This acute episode may escape the notice of the parents, and the cause may be obscured for a long period. Radiology is the primary means of confirming the diagnosis. However, it is seen that most foreign bodies are radioluscent. Chest roentgenograms are frequently normal if taken immediately after the foreign body inhalation and findings of obstructive emphysema and atelectasis are not always present. Great advances in endoscopic and anaesthetic techniques have greatly reduced the trauma and complications previously associated with endoscopic removal of tracheobronchial foreign bodies.

\section{Materials and Methods}

Thirty patients with foreign body inhalation treated in the department of Otorhinolaryngology and Head and Neck Surgery, B.J.Medical College (Civil Hospital), Ahmedabad, during 2001-2003, were included in this study. Their ages ranged from 6 months to 10 years. Diagnosis was made on the basis of history, clinical findings (Table 1), radiological evaluation (Table 2) and strong index of suspicion. In all cases foreign bodies were extracted with rigid bronchoscope under general anaesthesia. 
Table 1 Symptoms and Signs

\begin{tabular}{lcc}
\hline & Number of cases & Percentage of cases \\
\hline Symptoms & 18 & 60 \\
Penetration syndrome & & 10 \\
Cough & 3 & 10 \\
Sudden paroxysms & 3 & 10 \\
Repeated paroxysms & 3 & 47 \\
Persistent irritating & 24 & 7 \\
Breathlessness & 2 & 47 \\
Fever & 14 & 7 \\
Wheezing & 2 & 5 \\
No symptoms & & 50 \\
Signs & 15 & 33.3 \\
Decreased or absent air entry & 10 & 50 \\
Decreased movement of chest wall & 15 & 17 \\
Stridor & 05 & 33.3 \\
Rhonchi & 10 & 10 \\
Asthmatoid wheeze & 03 & 67 \\
Crepitations & 20 & \\
Tachypnoea & &
\end{tabular}

Table 2 Radiological evaluation before bronchoscopy

\begin{tabular}{lcc}
\hline Findings & Number of cases & Percentage of cases \\
\hline Obstructive emphysema & 05 & 17 \\
Atelectasis & 02 & 07 \\
-partial & 03 & 10 \\
-complete & & \\
& & 10 \\
Compensatory emphysema of contralateral lung & 03 & 07 \\
Pneumonitis & 02 & 33 \\
Mediastinal shift & 10 & \\
\hline
\end{tabular}

\section{Results and Analysis}

Rigid bronchoscopy was performed in all the cases which had history of foreign body inhalation. Patients' were aged between 6 months to 10 years. Aspiration in less than three years of age is common because children are getting accustomed to solid food and practicing swallowing. They lack molar teeth, which leads to improper chewing. $80 \%$ of cases belonged to this age group. 21 cases $(70 \%)$ were males and 9 cases $(30 \%)$ were females. Majorities of cases (90\%) were from low socioeconomic status. $50 \%$ patients presented in the first 24 hours, $13 \%$ of children were admitted within two days of aspiration, while $30 \%$ were admitted after two days. The longest interval between aspiration and admission was 40 days in one case.
History of foreign body aspirations was obtained in 24 cases $(80 \%)$, whereas typical history was not available in 6 cases $(20 \%)$. The most frequent symptom was 'penetration syndrome'-defined as sudden onset of choking and intractable cough with or without vomiting followed by dyspnoea as seen in $60 \%$ of cases.

On radiological examination (x-ray chest, anteroposterior and lateral view), in $10 \%$ of cases foreign body was opaque and visible on radiographs, $50 \%$ children had no detectable radiological findings, while $40 \%$ children showed indirect radiological signs. About $17 \%$ of our patients showed air trapping on the affected lung as a result of valve mechanism caused by the foreign body (obstructive emphysema), 17\% of x-rays showed resorptional atelectasis of the parts blocked by the foreign body from air supply and $10 \%$ had a compensatory emphysema of the contralateral side of the lung 

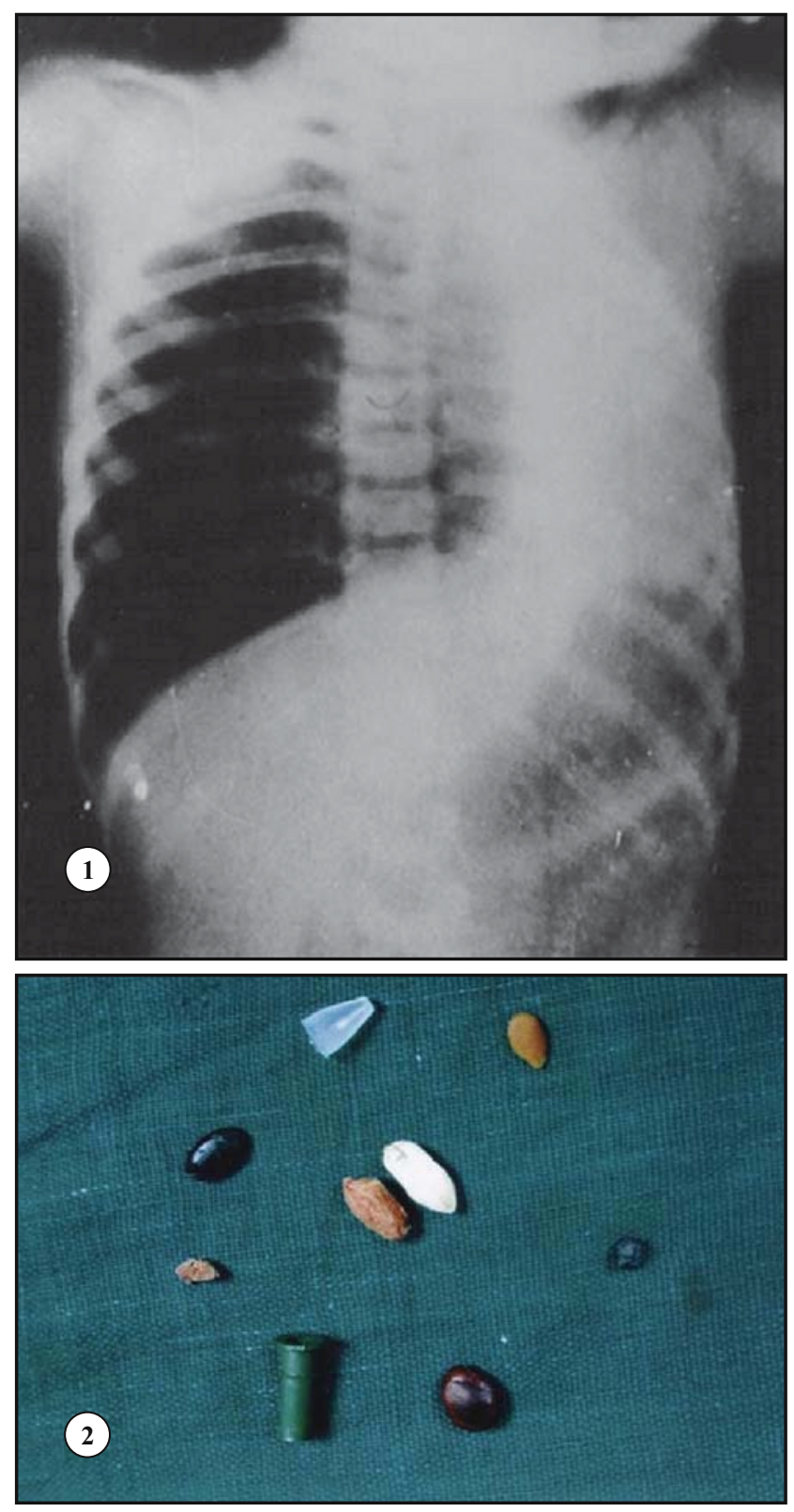

Fig. 1 X-ray showing complete collapse of left lung with compensatory emphysema of right Lung

Fig. 2 Various tracheobronchial foreign bodies
(Fig. 1). In $7 \%$ of cases signs of pneumonia were seen and $33 \%$ of x-rays showed a shift of the mediastinum. Fluoroscopy was performed on $50 \%$ of cases and $67 \%$ of the examination showed "swinging mediastinum"(HolzkmechtJacobson phenomenon).

Out of the 30 bronchoscopies performed, exogenous foreign bodies were removed in 29 cases while 1 case had mucous plug causing bronchial obstruction (endogenous). Out of the 29 foreign bodies (Fig. 2), majority (73\%) were of vegetative origin while only $23 \%$ had non-vegetative origin. Majority of vegetative foreign bodies were found in younger children ( $<3$ years), while non-vegetative foreign bodies were seen in older age group ( $>3$ years). The ingestion of groundnut accounted for the heighest incidence of foreign body aspiration (41\%) followed by grams, beetlenut, and amaliseed (14\% each). In majority of cases foreign body was found lodged in right bronchus (16 cases, 53\%) followed by trachea (09 cases, 30\%) and left bronchus (05 cases, 17\%). Following factors may take part for right side preponderance of foreign body lodgment: 1) its greater diameter; 2) the lesser angle of deviation of its axis from the tracheal axis; 3) the situation of carina to the left of the midline of trachea; and 4) the greater volume of air going into the right main bronchus on inspiration.

\section{Management}

Diagnosis was arrived in all cases after careful history taking, respiratory examination and investigation. The history of foreign body was available in 24 cases $(80 \%)$, radiological examination with positive findings in $50 \%$ of cases and positive clinical findings in $80 \%$ of cases. So, when we consider all these factors together for diagnostic purpose, accuracy will be $100 \%$ ( Table 3 ).

According to Mc Crae "there is no rule of thumb by which the presence of foreign body can be recognized, but there are general principles which assist greatly. Physical signs cannot determine the character of an opaque object as an x-ray can, but as a general rule, physical diagnosis should determine the presence of foreign body accurately". Bronchoscopy was carried out in all cases. Consent for bronchoscopy and simultaneous tracheostomy as and if needed (S.O.S.) was taken and risks and benefits were explained to the patients' relatives. Different and appropriate

Table 3 Accuracy rate

\begin{tabular}{|c|c|c|c|c|c|}
\hline \multicolumn{2}{|l|}{ Accuracy rate } & \multicolumn{2}{|c|}{ Clinical signs positive } & \multicolumn{2}{|c|}{ Radiological signs positive } \\
\hline \multicolumn{6}{|c|}{ With positive history of foreign body } \\
\hline No. Cases & Percentage $(\%)$ & No.of cases & Percentage $(\%)$ & No.of cases & Percentage $(\%)$ \\
\hline 24 & 80 & 21 & 70 & 12 & 40 \\
\hline \multicolumn{6}{|c|}{ With no history of foreign body } \\
\hline 06 & 20 & 03 & 10 & 03 & 10 \\
\hline
\end{tabular}


sized rigid bronchoscopes for the age of the patient were used. In $67 \%$ of cases, bronchoscopy with foreign body was done within 30 minutes. Only in 3 cases bronchoscopy extended to more than one hour. All the cases of vegetative foreign body of long duration, where foreign body had considerably swollen up were removed in pieces. Check bronchoscopy was done in all cases except one, where patient had to be intubated due to poor oxygen saturation. None of the patients had undergone tracheostomy procedure. In all the 30 cases, post-operative period was uneventful. $50 \%$ of our cases had mild fever during first 12 hours of post-bronchoscopy. There were no complications in post-operative period. Antibiotics, corticosteroids and bronchodilators were administered.

\section{Conclusion}

Though the human body is provided with a number of defense mechanisms to keep the airway free and clear of extraneous matter, foreign bodies frequently lodge in the airway of children. Children under 3 years of age are commonest victims $(80 \%)$. Males are more commonly affected than females (70:30). This can be explained by their being more active. Poor socio-economic status is contributory and neglect of children makes them more prone. History, symptoms and signs may give significant evidence of the presence of foreign body. Around $80 \%$ of cases could be diagnosed based on clinical evaluation. Radiological examinations are not confirmatory but contributory to the diagnosis. Non radio-opaque foreign bodies are more common $(90 \%)$, than radio-opaque $(10 \%)$. Vegetative foreign bodies are more common $(73 \%)$, than non-vegetative (27\%). A cough of unknown origin that resists therapy might prove, after detailed questioning of the parents, detailed examination and a chest $\mathrm{x}$-ray examination to be caused by a foreign body. Bronchoscopy is essential in all cases of tracheobronchial foreign bodies, known or suspected. A complete range of pediatric bronchoscopes together with different types of foreign body forceps and other accessories greatly influence the outcome of the procedure and a good set-up is mandatory to ensure comfortable and confident endoscopy and optimal safety for the patients. Bronchoscopy done under general anaesthesia and patient ventilating using a jet ventilator is a very safe and effective technique. A second look into the airway, soon after removal of foreign body is emphasized.

\section{References}

1. Shivakumar AM (2003) Tracheobronchial foreign bodies, Indian journal of Paediatrics Vol. 70, 793-797

2. Mathur NN (2003) Tapaswami Pradhan: Rigid paediatric bronchoscopy for bronchial foreign bodies with or without Hopkins telescope, Indian Paediatrics 40:761765

3. Martin E (2002) Warschawsky: eMedicine Vol 3 (through WebMD)

4. Skoulakis CE (2000) Bronchoscopy for foreign body removal in children. A review and analysis of 210 cases. International Journal of Paediatric Otolaryngology 53(2): 143-148

5. Farhad B (1999) Tracheobronchial foreign bodies presentation and management in children and adults. Chest 115: $1357-1362$

6. Zerella JT (1998) Foreign body aspiration in children: Value of radiography and complications of bronchoscopy. Journal of Paediatric surgery 33(11):1651-1654

7. Johnson DG, (1998) Condon VR: Foreign bodies in the paediatric patients.Current problems in Surgery 35:275-279

8. Kay HS, Th. Zimmermann MD (1990) Tracheobronchial aspiration of foreign bodies in children: A Study of 94 casesLaryngoscope 100 (5):525-530 